\title{
Archéopages
}

Archéopages

Archéologie et société

Hors-série 1 | 2008

Construction $^{s}$ de l'archéologie

\section{En marge des femmes : la société contre le désir des hommes}

Pierre Lemonnier

\section{(2) OpenEdition}

1 Journals

Édition électronique

URL : https://journals.openedition.org/archeopages/865

DOI : 10.4000/archeopages.865

ISSN : 2269-9872

Éditeur

INRAP - Institut national de recherches archéologiques préventives

Édition imprimée

Date de publication : 1 février 2008

Pagination : 90-94

ISSN : 1622-8545

\section{Référence électronique}

Pierre Lemonnier, «En marge des femmes : la société contre le désir des hommes », Archéopages [En ligne], Hors-série 1 | 2008, mis en ligne le 01 février 2008, consulté le 23 février 2023. URL : http:// journals.openedition.org/archeopages/865 ; DOI : https://doi.org/10.4000/archeopages.865

Tous droits réservés 


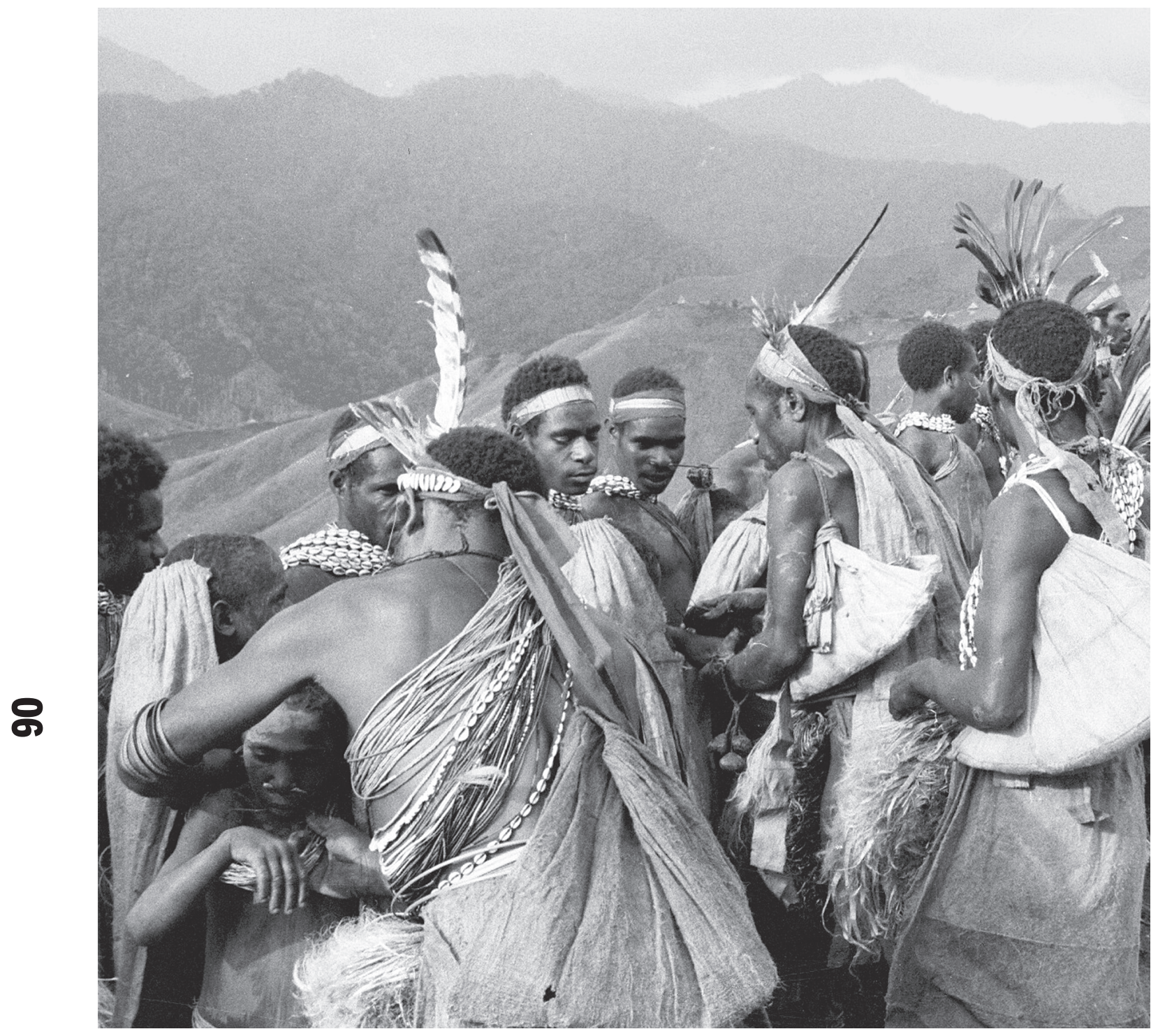

[Fig.1] Chez les Baruya de Nouvelle-

Guinée, les maîtres des initiations

perdent presque tout pouvoir une fois

les rites masculins terminés. 
Richard H., VignOt A. (dir.) 2002 : Équilibres et ruptures dans les écosystèmes depuis 20 ooo ans en Europe de l'ouest, Colloque international, Besançon, septembre 200o, Paris, Les Belles Lettres, p.191-203. (Annales littéraires de Franche-Comté, série Environnement, Société et Archéologie 3).

UNESCO 2003 : Paris: Convention pour la sauvegarde du patrimoine culturel immatériel, 17 octobre 2003 ; http://www.unesco.org/ culture/ich/index.php?pg=00022

VAN Der Leeuw S., Favory F., Fiches J.-L. (dir.) 2003: Archéologie et systèmes socio-environnementaux: Études multiscalaires sur la vallée du Rhône dans le programme Archeomedes, Paris, CNRS (monographie du Cra).

\section{En marge des femmes : la société contre le désir des hommes}

\author{
Pierre Lemonnier \\ CNRS-Credo
}

La question n'est donc plus: comment le pouvoir émerge-t-il? Mais plutôt: pourquoi les mécanismes de contrôle d'une émergence excessive du pouvoir font-ils parfois défaut? Jean-Paul Demoule (2007, p. 88)

$\mathbf{P}$ lus discret sur les conditions d'apparition de l'État (1974, p. 161-186) que dans son ahurissante détestation des marxistes (1980 [1978]), Pierre Clastres défendit, dès 1969, l'idée que les sociétés « ont très tôt pressenti que la transcendance du pouvoir recèle pour le groupe un risque mortel» (1974 [1969], p. 40). Selon l'auteur de La Société contre l'État, ce fut la mise en place du couple coercition-subordination, cette « essence du pouvoir partout et toujours » (ibid., p. 12, souligné par Clastres), qui conduisit aux «sociétés historiques [...] qui portent en elles la cause de l'innovation, du changement, de l'historicité » (ibid., p. 22, souligné par Clastres). Appuyée sur le solide portrait d'un leader politique amazonien dépourvu d'autorité, cette théorie d'une limitation du pouvoir par les sociétés locales n'a pas manqué de faire école. Chez Marshal Sahlins (1963), sans doute, dont le big man mélanésien ressemble comme un frère au chef «contraint, sous peine de se voir rapidement abandonné par la plupart de ses gens, de tenter de satisfaire leur demande»(Clastres 1974, p. 35), et jusqu'à Jean-Paul Demoule, qui consacre trois paragraphes de son dernier ouvrage aux divers moyens, pour une société, de «se prémunir contre la montée de pouvoirs trop forts» (2007, p. 89).

La société, bien entendu, n'est contre rien du tout car elle n'existe qu'à travers des pensées individuelles, fussent-elles inscrites en institutions ou mises en gestes et en objets. En revanche, la recherche de l'équité conduit parfois les humains à créer des enchaînements de pratiques dont les effets sociaux et matériels, aussi inattendus qu'incontrôlables, sont à l'opposé de l'objectif recherché. C'est cette dimension inintentionnelle et paradoxale des logiques sociales que j'aborderai ici, à partir de l'exemple de sociétés de Mélanésie.

Fanatiquement égaux. À côté de "chefs » contrôlant la vie de tous à coups d'interdits et ayant jadis droit de vie et de mort sur leurs sujets, la Mélanésie comporte (-ait) nombre de personnages politiques auxquels les observations de Clastres sur l'équité dans les sociétés préindustrielles s'appliquent spectaculairement. Des Anga de Papouasie Nouvelle-Guinée, on dirait certainement que «l'idée de donner un ordre ou d'avoir à obéir» leur est «quelque chose de tout à fait étranger» (1974, p.11-12). Et l'autorité temporaire de leurs grands guerriers s'accorde tout autant à cette constatation que «le modèle du pouvoir coercitif n'est [...] accepté qu'en des occasions exceptionnelles, lorsque le groupe est confronté à une menace extérieure » (ibid., p. 27).

Surtout, beaucoup de sociétés mélanésiennes ont elles aussi ce «goût de l'égalité» repéré par Clastres (ibid., p. 26) et déploient des efforts spectaculaires pour défendre la parité des personnes et des échanges. Cela était (ou reste) particulièrement explicite sur la côte sud de la grande île, où s'appliquait (s'applique) un strict principe d'équivalence (en nature et en quantité) dans les domaines les plus variés: se marier par échange de sœurs (réelles ou classificatoires), tuer pour venger un assassinat ou une perte à la guerre, infliger à un agresseur la même blessure que celle qu'il a causée, payer une contremagie le prix qu'a coûté l'acte de sorcellerie qu'elle est censée annuler, échanger une femme contre une femme, divorcer parce que la sœur que l'on a donnée en échange de sa propre femme se sépare de son mari, prêter son épouse contre celle de son amant d'une nuit, rendre autant que l'on a reçu, etc. (Lemonnier 1993).

\section{La société contre les idées reçues : exploitation} et despotisme chez les big men. Nombre de groupes de Mélanésie n'en ont (ou n'en avaient) pas moins des leaders dont le statut social est bien plus qu' «enviable» (Clastres 1974, p. 35) et comporte la faculté de se faire obéir et d'exploiter les autres, et d'abord leur travail, y compris les big men, qui constituent à juste titre la version mélanésienne de ces «fœetus culturels du pouvoir [...] voués à périr» (ibid., p.17). Bien que présentés comme des personnages politiques situés à l'écart du chemin- qui-mèneà-la-chefferie-voire-à-l'État grâce à des mécanismes sociologiques autorégulateurs s'opposant à l'accumulation de pouvoir, les big men ne se privaient pas d'exploiter autrui, comme l'indique le triste sort de ces rubbish men décrits par Meggit (1977, p.190-191) chez les Mae-Enga. Lors d'une redistribution de biens par un big man, ces «pas grand-chose»-qui ont aidé le haut personnage à rassembler les biens (porcs, coquillages) offerts par lui au nom de tous lors d'un précédent échange compétitif viennent demander leur part. Mais, loin de voir leur revendication satisfaite, ils sont éconduits puis frappés par d'autres protégés du big man. Non seulement le big man mélanésien prospère en convainquant les autres de lui fournir gratuitement leur travail (pour élever les porcs 
qu'il offrira en leur nom), mais il peut marginalement exploiter par la force le labeur de quelques malheureux vivant à la marge de la société. À défaut de sociétés inventant l'État, la Mélanésie ne manque donc pas de groupes revendiquant un égalitarisme forcené, tout en connaissant (contre l'avis de Clastres) un amer avant-goût du couple commandement-obéissance et de l'exploitation du travail d'autrui.

Quels mécanismes sociologiques, conditions, pratiques, biais des systèmes de pensée expliquent cette contradiction? La moitié de (la fourberie) de l'Histoire est bien connue: l'approvisionnement des échanges cérémoniels de richesses, dans l'organisation desquels brille un big man, est fondé sur un système où celui qui élève des porcs pour autrui est payé pour sa peine en recevant du prestige et des porcelets... qui sont le fruit de son propre travail (Lemonnier 1990, p. 29-35). L'autre mâchoire du piège inégalitaire a quelque chose à voir avec un désir de biens matériels, celui-là même que Clastres (1974, p. 164-168) jugeait incompatible avec le «refus du travail» caractérisant, parait-il, les sociétés "primitives». Je me propose de montrer que cet attrait pour les richesses est une conséquence directe de ce désir d'échanger de l'identique à l'identique, en l'occurrence des femmes contre des femmes, mais des femmes jugées de même valeur. Et d'illustrer l'une de ces farces que les logiques inintentionnelles jouent aux hommes et aux sociétés.

Quand les sœurs ne se valent pas. L'ethnographie de la Mélanésie indique que deux grains de sable peuvent se loger dans la mécanique bien huilée de l'échange de sœurs. En l'absence d'une telle parente à échanger, les Keraki de la côte sud de la grande île offraient des biens précieux pour se procurer la «sœur» qu'il leur fallait céder pour se marier eux-mêmes (Williams 1936, p.128, 139). Dans les mêmes circonstances, les Jaqaj donnaient à leurs affins des richesses considérées comme un versement provisoire, dans l'attente qu'une femme soit disponible pour être rendue. Si tel était le cas, ce dépôt de garantie était restitué. Quant aux Kimam, ils versaient une sorte de dédommagement pour "rupture de contrat» lorsque, pour quelque raison, la femme attendue en échange d'une épouse n'était finalement pas donnée (Serpenti 1977, p.129-130).

Lautre circonstance - qui voit des sociétés injecter farouchement des biens précieux dans le mariage par échange des sours - résulte du désir d'échanger des femmes aussi identiques que possible. Tel est le cas des Baruya, qui donnent alors des richesses pour compenser une inégalité constatée entre les femmes échangées. Deux ou trois générations après un mariage pour lequel aucune femme n'a été rendue (alors même que de nombreuses filles et petites-filles en sont nées) ou bien si l'une des deux femmes a mis au monde un nombre d'enfants beaucoup plus élevé que l'autre, les descendants des parents paternels et maternels de celle-ci réclament une compensation au lignage preneur. Chez les Maring, cette recherche de l'équité va jusqu’à anticiper une possible disparité dans le mariage, et l'échange direct de sœurs s'accompagne du versement d'une compensation matrimoniale, car, dit-on, «la valeur de chaque femme [son ardeur au travail ou le nombre d'enfants qu'elle mettra au monde] est encore inconnue, et il n'y a donc aucun moyen de savoir si l'échange est équivalent » (Lipuma 1988, p. 150 sq., 165). Pour une raison identique, chez les Kiwai de la côte sud de lîle, un homme dont l'épouse mettait au monde de nombreux enfants offrait des richesses à ses beaux-parents «en reconnaissance des avantages qu'elle apportait à son 〈sang \» (Landtman 1927, p. 244-245).

Qu'il s'agisse d'établir une parité impossible lorsqu'il n'existe pas de «sœur » à rendre, de prévoir la symétrie future d'un échange de femmes ou de corriger un déséquilibre constaté après des générations, ces dédommagements répondent à une recherche constante d'égalité dans le domaine du mariage qui est en accord avec la forme générale des échanges dans les sociétés en question. C'est pour souligner ou rétablir une équivalence «en nature » entre les deux femmes que l'on introduit une non-équivalence partielle dans les termes de l'échange de mariage (une femme d'un côté, des objets de l'autre). Or, l'usage de richesses dans des unions par échange direct de femmes est un pas vers l'institution du mariage avec compensation matrimoniale - un type d'union qui s'accompagne de l'inégalité durable de ceux qui échangent (preneurs et donneurs étant dans une relation asymétrique), car, dans la majorité des cas, une femme ne sera jamais « correctement » compensée par des richesses, et il faut continuellement faire des dons à ses affins ou aux maternels des enfants. Les Mélanésiens ne s'y sont pas trompés, qui ont étroitement associé au mariage avec d'anciens ennemis les échanges compétitifs de richesses par lesquels ils remplacent temporairement la guerre... comme Lévi-Strauss (1943) l'indiquait jadis à propos de l'Amazonie.

\section{Limiter la production de richesses n'implique} pas l'absence de coercition. Clastres aurait peut-être fait remarquer qu'aucune de ces sociétés (où le don de biens précieux vient compenser un déficit d'égalité entre des femmes échangées dans le cadre du mariage) n'a développé de système politique lié à la circulation de ces richesses, car elles ne sont justement pas des sociétés à big men. On ajoutera même que, tant chez les sociétés de la côte sud de la grande île que chez les Anga, le cloisonnement des échanges est un frein à cette généralisation de l'usage des richesses qui caractérise les groupes à big men D'abord, à l'inverse de ce qui se passe chez les big men où un même type de bien (coquillages, porcs) peut servir dans toutes sortes d'échanges, on peut réserver l'usage de certaines richesses à autant de types d'échange bien précis (mariage, relation avec les affins, compensation pour homicide, etc.). 
Ensuite, d'autres moyens que le don de richesses en l'occurrence le don d'enfants et l'offre temporaire des services sexuels d'une femme permettent de compenser une vie, un tort, du travail ou un service (Lemonnier 1999).

À défaut de fournir une épouse en échange d'une femme donnée en mariage, les Ilahita Arapesh offrent ainsi un enfant né du mariage. Chez les Maring, lorsqu'un échange de sœurs est « techniquement» impossible et lorsqu'il n'est pas versé de bridewealth d'attente, une fille née du mariage se rend chez son oncle utérin, qui la donne en mariage pour obtenir lui-même une compensation matrimoniale qu'il réutilisera. Quant au prêt/don non réciproque des services sexuels d'une femme (d'une épouse) comme dédommagement dans divers contextes concernant des services et des torts ordinaires, il s'observait dans le sud de la Nouvelle-Guinée, précisément là où les biens précieux s'infiltrent à la périphérie des unions par échange de sœurs. Par exemple, en offrant son épouse, un Kolopom, un Kiwai ou un Marind-anim indemnisait la victime d'un vol qu'il avait commis, rémunérait les services d'un tueur, d'un magicien ou d'un sorcier, voire une aide dans le travail ou le prêt d'un outil.

Il est donc incontestable que ces sociétés (où j'ai signalé le rôle institutionnalisé de richesses dans un monde pourtant obsédé par l'égalité) limitaient l'usage de celles-ci aux compensations pour la vie et la mort (mariage et homicide), c'est-à-dire au devenir des personnes, tandis que le prêt de femmes, considérées comme objets de plaisir, tenait lieu de dédommagement dans d'autres contextes. Un clastrien pourrait voir là une volonté de limiter la place des richesses, voire d'éviter le franchissement de ce seuil que constitue, en Mélanésie, l'existence d'une sphère unique d'échanges, en ne considérant pas le porc domestique comme un moyen légitime de compenser pour la vie humaine (mariage et homicide).

On est donc loin des big men, qui eux-mêmes sont loin des chefs, de l'État, etc. Il n'en demeure pas moins que, si ces sociétés sont pour l'égalité, leurs membres la recherchent d'une manière qui porte en germe l'essor de la production de biens, le travail aliéné et l'exploitation d'autrui, et ce, du simple fait de l'introduction de biens précieux dans ce domaine éminemment concerné par la circulation de la vie humaine qu'est le mariage. Faire intervenir des richesses dans des compensations pour la vie - pour des femmes vivantes qui donneront la vie -, c'est admettre et permettre qu'une partie de ce qui est échangé dans un mariage sorte de la sphère de la réciprocité en nature. L'ethnographie montre que c'est également imaginer que les tués peuvent être compensés par des biens, et non par d'autres tués (Lemonnier 1993). Or, du fait du recours au mariage pour établir ou maintenir la paix et dans un contexte culturel où la compétition économique peut provisoirement remplacer la guerre, ce recours aux richesses dans la compensation de la vie est la condition sine qua non de la mise en place des systèmes d'échanges cérémoniels de richesses où brillent les bigmen, avec les inégalités patentes que j’ai rappelées.

\section{Vers quelle étagère? Des traces pour}

l'archéologue? C'est paradoxalement dans le but de maintenir, de prévoir ou de rétablir une rigoureuse parité entre les individus et les groupes que des sociétés de Nouvelle-Guinée introduisent, sous la forme de richesses se substituant partiellement aux personnes, les instruments qui sont au fondement d'une ébauche d'inégalité. C'est le désir d'égalité des femmes que les hommes échangent qui ouvre la porte à des systèmes sociaux moins égalitaires, voire franchement inégalitaires.

La logique dont j'ai essayé de montrer les ruses ne relève ni d'une «métaphysique finaliste », justement mise en cause par Clastres, ni des modèles inconscients qu'élabore le groupe et auxquels il rapportait in fine le non-pouvoir de la fonction politique en Amazonie. Elle relève de ce qu'il considérait, lui, comme impossible: «le 〈dérapage > d'un système malgré lui démenti par un résultat que le groupe serait incapable de corriger» (Clastres 1974, p. 38-39). C'est hors de la conscience des hommes et des femmes et sans qu'intervienne aucun modèle politique inventé par une société qui «sait, par nature [sic], que la violence est l'essence du pouvoir» (ibid., p.136) - que s'accumulent les conditions d'une transformation menant à l'intensification de la production et de l'exploitation de malheureux laissés pour compte. Ici, nulle institution politique n'est en cause, mais seulement une volonté de paix entre des beaux-frères et des familles qui échangent des sœurs en annulant les différences de celles-ci.

Avant l'État, il y a quelque chose comme les classes et les castes, en tout cas une différenciation des hommes (et des femmes) qui passe par des richesses dont les archéologues savent repérer les traces : tombes à char, morts d'accompagnement, parures, grandes maisons, grands domaines, matières et objets rares. À défaut d'improbables traces du « refus du travail» ou du «sens du loisir» (ibid., p. 167-168, 173), il n'est peut-être pas impossible de repérer aussi ces objets précieux qui annoncent autant le désir d'égalité que la sujétion, en se tournant vers la fouille et les cabanes de chantier. Mais vers quelle étagère?

Clastres P. 1969: «Copernic et les sauvages», Critique, $\mathrm{n}^{\circ}$ 270, repris in Clastres 1974, p. 7-24.

Clastres P. 1974: La Société contre l'État, Paris, Éditions de Minuit.

Clastres P. 1980: Recherches d'anthropologie politique, Paris, Le Seuil.

Demoule J.-P. 2007: La Révolution néolithique en France, Paris, La Découverte.

Landtman G. 1927: The Kiwai Papuans of British New Guinea, Londres, Macmillan and Co.

Lemonnier P. 1990 : Guerres et festins. Paix, échanges et compétition dans les Hautes Terres de Nouvelle-Guinée, Paris, Éditions de la Maison des sciences de l'homme.

LEMONNIER P. 1993: «Le porc comme substitut de vie: formes de compensation et échanges en Nouvelle-Guinée», Social Anthropology, nº 1 , p. 33-55. 
LEMONNIER P. 1999: «Femmes et richesses en Nouvelle-Guinée», in P.Descola, J.Hamel, P. Lemonnier (dir), La Production du social. Autour de Maurice Godelier, Paris, Fayard, p. 315-332.

LÉVI-STRAUSS Cl. 1943: " Guerre et commerce chez les Indiens de l'Amérique du Sud », Renaissance, nº 1, p.122-139.

Lipuma E. 1988: The Gift of Kinship, Cambridge, Cambridge University Press.

Meggitt M. 1977: Blood is their Argument, Palo Alto, Mayfield Publishing Company.

SAHLINS M. 1963 : «Poor man, rich man, big-man, chief: political types in Melanesia and Polynesia ", Comparative studies in society and history, $\mathrm{n}^{\circ} 5$ (3), p. 285-303.

SERPENTI L. 1977: Cultivators in the Swamps. Social structure and horticulture in a New Guinea society (Frederik-Hendrik Island West New Guinea), Assen, Amsterdam, van Gorgum.

Williams F. E. 1936: Papuans of the Trans-Fly, Oxford, Clarendon Press.

\section{Archéologie et langage : éloge du scepticisme}

Colin Renfrew

McDonald Institute for Archaeological Research, Cambridge

$\mathrm{L}$ es spécialistes des études indo-européennes des années 1960-1970 affirmaient (ou présumaient) qu'au début de l'âge du Bronze, en Europe centrale et occidentale, une «arrivée » d'Indo-Européens avait eu lieu, de même qu'à une époque indéterminée de lâge du Fer avait eu lieu une «arrivée » des Celtes, et que durant l'âge du Bronze ou vers sa fin avait eu lieu également une «arrivée» des Grecs. Chacun de ces événements avait été d'abord avancé ou supposé sur une base linguistique, à charge pour l'archéologie d'en apporter ensuite la preuve.

Je souhaiterais souligner ici la position courageuse - solitaire parfois - de Jean-Paul Demoule $(1980,1999)$ sur les langues et les populations indo-européennes. C'est en archéologue qu'il aborda l'enjeu d'une possible réconciliation des données linguistiques et archéologiques en Europe. Il a clairement perçu que l'ensemble des opinions qui prévalaient dans les années 1960 et 1970, à l'instar de la plupart des précédentes, étaient sujettes à controverse car elles se fondaient sur des généralisations et des hypothèses sur les migrations qui nétaient plus appuyées par les preuves archéologiques. À l'époque, de son côté comme du mien, nous pouvions observer que les équations trop faciles entre des langues et des «cultures» archéologiques spécifiques, d'une part, et des «peuples» hypothétiques (souvent fictifs), d'autre part, qui étaient supposés avoir parlé ces langues et avoir créé ces cultures, présentaient d'irrémédiables défaillances.

Ma propre attitude à l'égard de cette constatation était peut-être moins politique, moins socialement engagée que la sienne (bien que nous partagions la même antipathie pour l'idéologie nazie, le racisme, et plus particulièrement l'antisémitisme); il en sait plus que moi sur l'histoire intellectuelle du $\mathrm{xx}^{\mathrm{e}}$ siècle et peut-être aussi sur les sympathies de certains intellectuels renommés, comme Georges Dumézil, dont nous rejetons d'un commun accord les synthèses. En ce qui me concerne, mon doute initial à propos des études indo-européennes des années 1960 et 1970 ne reposait pas tant sur une position politique que sur l'influence constante qu'elles exerçaient sur la reconstruction archéologique. En dépit de l'affection et du respect que je voue à Marija Gimbutas, sa reformulation de la théorie avancée un demi-siècle auparavant par Gordon Childe (qui n'était lui-même pas le premier) à propos d'invasions de guerriers nomades montés à cheval en provenance des steppes orientales m'a toujours paru manquer de substance archéologique. Or, cette hypothèse «kourgane» jouit encore plus ou moins des faveurs de la majorité des linguistes historiques et d'archéologues aussi réputés que James Mallory. Jean-Paul Demoule et moi-même pourrions probablement exprimer une critique identique à l'encontre de cette théorie - critique d'autant plus fondée que nous possédons à présent les preuves que le cheval ne fut utilisé pour la première fois dans des opérations militaires pour tracter des chars quà partir de 2000 environ avant notre ère, et qu'il y a peu d'éléments prouvant l'apparition d'une cavalerie montée avant le premier millénaire précédant notre ère.

Ces constatations ont amené Demoule à faire preuve d'audace et à rejeter l'intégralité du concept d'une langue proto-indo-européenne, une Ursprache et, partant, d'une Urheimat. Rares sont ceux qui ont eu le courage d'adopter une position aussi radicale. Contester la totalité des études indo-européennes demande un sacré culot! Des préoccupations similaires mont encouragé à réexaminer le concept indo-européen dans son ensemble, bien qu'avec une attitude moins radicale. J'étais surpris par le consensus apparent entre les linguistes selon lequel les relations entre les langues qualifiées aujourd'hui d'«indo-européennes» ne pourraient s'expliquer que par une origine commune, une Ursprache, autrement dit une prétendue langue «proto-indoeuropéenne », comme l'avait suggéré sir William Jones il y a quelque deux cent cinquante ans. Cela impliquerait une sorte d'arbre généalogique linguistique, calqué sur le modèle proposé par A. Schleicher en 1863, suivant les principes de Charles Darwin. Dans mon ouvrage intitulé Archaeology and Language (L'Énigme indoeuropéenne: archéologie et langage), j’ai souligné les autres possibilités proposées par la théorie des «vagues » de Johannes Schmidt, qui, dès 1875, démontrait comment des langues voisines pouvaient s'influencer les unes les autres et se renouveler au moyen d'emprunts. Mais peu de linguistes ont osé suggérer que les langues appartenant à ce que l'on appelle généralement une «famille linguistique» pouvaient devoir l'ensemble de leurs traits communs familiaux à ce processus d'emprunts, comme l'a fait Demoule. Du reste, le seul linguiste à avoir appliqué cette hypothèse à la famille des langues indo-européennes, pour autant que je sache, fut Nicolas Troubetskoï en 1939. Il y a quelques années, quand j'ai mentionné ses travaux en Australie, sa ligne de pensée fut reprise avec énergie par Robert Dixon (1997), mais pas 